\title{
Secondary meningioma in a long-term survivor of atypical teratoid/rhabdoid tumour with a germline INI1 mutation
}

\author{
A. C. J. Ammerlaan • M. P. W. A. Houben • \\ C. C. Tijssen • P. Wesseling • T. J. M. Hulsebos
}

Received: 21 December 2007 /Published online: 31 January 2008

(C) The Author(s) 2008

\begin{abstract}
Objective We report on a patient who developed a meningioma more than two decades after removal at a young age of an atypical teratoid/rhabdoid tumour (AT/RT), which was due to a germline INI1 mutation, and radio- and chemotherapy.

Materials and methods We present genetic evidence that the meningioma is not a recurrence or metastasis of the AT/ RT and not due to the INII mutation, but is a radiationinduced tumour.

Conclusion This is the first case illustrating that improved survival of young patients with an AT/RT after aggressive treatment may be gained at the cost of an increased risk for the development of radiation-induced, non-INI1-related tumours.
\end{abstract}

Keywords Meningioma $\cdot$ Radiation therapy

Atypical teratoid/rhabdoid tumour - Genetic predisposition . INII

\section{Introduction}

We previously reported on a family in which four cousins developed an atypical teratoid/rhabdoid tumour (AT/RT) at

A. C. J. Ammerlaan • T. J. M. Hulsebos $(\bowtie)$

Department of Neurogenetics, Academic Medical Center,

Meibergdreef 9 ,

1105 AZ Amsterdam, The Netherlands

e-mail: t.j.hulsebos@amc.uva.nl

M. P. W. A. Houben • C. C. Tijssen

Department of Neurology, St. Elisabeth Hospital,

Tilburg, The Netherlands

\section{P. Wesseling}

Department of Pathology,

Nijmegen Center for Molecular Life Sciences,

Radboud University Nijmegen Medical Centre,

Nijmegen, The Netherlands young age, due to inheritance of a germline $\mathrm{G}>\mathrm{A}$ mutation in the donor splice site of exon 4 of the tumour suppressor gene INII [2]. More than 20 years after resection of the AT/ $\mathrm{RT}$, the oldest of the four cousins developed an intracranial meningioma and, after an additional year, a myoepithelioma of the lip. In addition to the constitutional INII mutation and loss of the normal INII allele, we detected an identical rearrangement in the $N F 2$ gene, not affecting its coding regions, in the AT/RT and myoepithelioma of the patient and concluded that they both originated from a common precursor cell. However, the rearrangement in $N F 2$ and loss of the normal INII allele could not be demonstrated in the meningioma. Despite this, the development of the meningioma may be the consequence of the presence of the constitutional INI1 mutation, because mutations in this gene have been identified in meningiomas [8]. Alternatively, the meningioma could be induced by the radiation therapy, which our patient received after removal of the AT/RT and which is a well-known consequence of this treatment [1]. We investigated the meningioma for the presence of genetic changes reported in radiation-induced meningiomas and performed a comparative loss of heterozygosity (LOH) analysis to demonstrate that such radiationinduced genetic changes are absent in the AT/RT and myoepithelioma of our patient.

\section{Materials and methods}

Tumour samples and DNA isolation

DNA from the AT/RT and the myoepithelioma was isolated from formalin-fixed and paraffin-embedded tumour material, and DNA from the meningioma was isolated from a fresh frozen tumour sample using commercially available kits (Qiagen, Venlo, The Netherlands). 
SNP analysis

A genome-wide single nucleotide polymorphism (SNP) analysis of normal and meningioma DNA was conducted with the Affymetrix GeneChip Human Mapping $10 \mathrm{~K}$ Array Xba 142 2.0. The array contains 10,204 SNPs distributed across the genome. The GeneChips were processed by an authorised Affymetrix Service Provider using standard Affymetrix protocols (Service XS, Leiden, The Netherlands). LOH regions were identified by comparing the SNP profiles of normal and meningioma DNA and scoring heterozygous SNP markers (AB) in the normal DNA that were reduced to homozygosity (AA or $\mathrm{BB})$ in the meningioma DNA. Intervals were determined on the basis of locations of SNP markers in the UCSC Genome Browser, March 2006 Build, at http://genome. ucsc.edu/.

\section{$\mathrm{LOH}$ analysis}

Loss of heterozygosity ( $\mathrm{LOH}$ ) analysis was performed with microsatellite markers as described previously [3] using primer sequences and conditions for polymerase chain reaction given by The Genome Database at http://www. gdb.org/. Because of rather extensive degradation of DNA extracted from the AT/RT, only markers generating PCR fragments smaller than $150 \mathrm{bp}$ were used.

\section{Case report}

History

Our patient was diagnosed with a tumour in the fourth ventricle and right cerebellar hemisphere at the age of 4.5 years. Resection of the tumour was incomplete. The tumour was originally classified as anaplastic ependymoma WHO grade III, but upon extensive genetic analysis and histopathological re-evaluation, reclassified as AT/RT [2]. He received adjuvant chemotherapy (methotrexate, vincristine and prednisolone) followed by craniospinal radiotherapy (3,300 cGy in 22 doses) with a boost of 2,100 cGy on the location of the tumour. Fifteen years later, a brain CT without contrast showed no signs of recurrent disease or of other new pathology. Almost 6 years later, 20.5 years after initial diagnosis, he presented again with bifrontal headaches, increasing ataxic gait disturbances and fatigue. CT examination showed a large isodens space-occupying lesion in the left temporal region with oedema, midline shift and contrast enhancement. One year after resection of this tumour, he developed a myoepithelioma of the upper lip. The patient is alive at 29 years of age.
Table $1 \mathrm{LOH}$ regions in the meningioma, as determined by SNP analysis

\begin{tabular}{ll}
\hline Chromosome region & Exact interval $(\mathrm{Mb})$ \\
\hline 1pter-p34.3 & $0-38.90$ \\
1p33-p33 & $46.99-51.09$ \\
1p32.2-1p31.3 & $58.19-65.50$ \\
2pter-p12 & $0-79.28$ \\
3p26.1-cen & $6.11-88.84$ \\
7q36.1-qter & $150.04-158.66$ \\
16pter-p12.1 & $0-22.75$ \\
16q23.1-q23.3 & $73.46-81.33$ \\
17cen-q21.31 & $23.09-40.35$ \\
19 pter-p13.2 & $0-8.83$ \\
\hline
\end{tabular}

Histological findings

Histopathological examination of the left temporal tumour revealed an atypical meningothelial meningioma WHO grade II with many vessels and up to six mitoses per $2 \mathrm{~mm}^{2}$.

SNP and LOH analysis Radiation-induced meningiomas usually display a complex karyotype [1]. To determine the genetic changes, we performed a genome-wide scan by SNP analysis of the meningioma and corresponding normal DNA. The identified chromosomal regions displaying $\mathrm{LOH}$ are listed in Table 1. Next, we tested several markers from within each of the five largest deleted regions by conven-
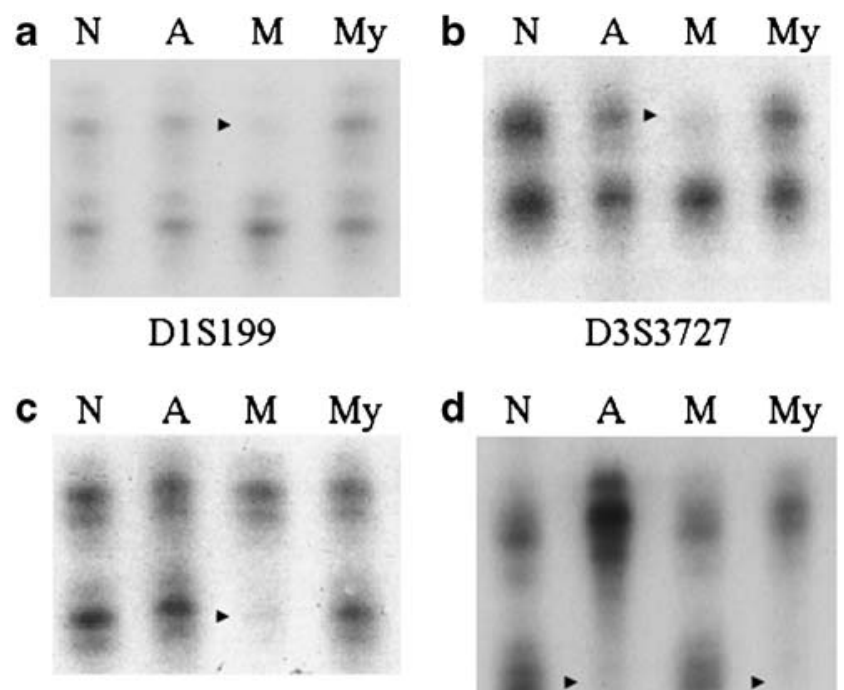

D17S1872

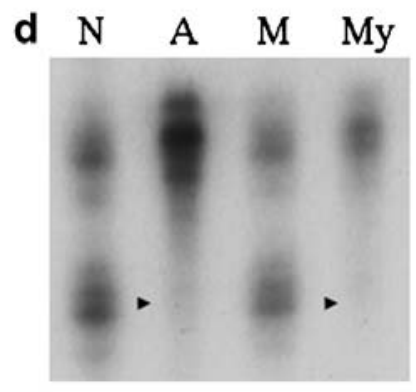

$\mathrm{D} 22 \mathrm{~S} 430$

Fig. 1 LOH analysis using markers from chromosome regions a 1pter-p34.3 (D1S199), b 3p26.1-cen (D3S3727), c 17qcen-q21.31 $(D 17 S 1872)$ and d 22q $(D 22 S 430)$ of AT/RT $(A)$, meningioma $(M)$ and myoepithelioma $(M y)$ DNAs of the patient. $N$ denotes normal DNA derived from the patient's blood leucocytes. Arrowhead indicates position of lost allele 
tional microsatellite analysis to determine their $\mathrm{LOH}$ status in the meningioma and in the AT/RT and myoepithelioma. Presence of $\mathrm{LOH}$ in the meningioma, but absence of $\mathrm{LOH}$ in $\mathrm{AT} / \mathrm{RT}$ and myoepithelioma was found for D1S199 in 1pterp34.3 (Fig. 1a), D2S327 in 2pter-p12, D3S3727 in 3p26. 1-cen (Fig. 1b), D16S3020 in 16pter-p12.1 and D17S1872 in 17qcen-q21.31 (Fig. 1c). In contrast, as determined previously [2] and exemplified by marker D22S430 in Fig. 1d, there was LOH for chromosome 22 markers in the AT/RT and myoepithelioma, but not in the meningioma.

\section{Discussion}

In a previous study, we provided evidence for the involvement of INII, by constitutional mutation and independent somatic loss, in the development of both the AT/RT and the myoepithelioma of our patient. Moreover, we could demonstrate that these tumours originated from an identical precursor cell [2]. Although involvement of INI1 has been described in sporadic meningiomas [8], until now meningiomas have not been reported to occur as a consequence of the inheritance of an INII mutation. However, this might be due to the extreme rarity of families with an inherited INII mutation, with only four published cases so far $[2,5,6,11]$. In this context, it is interesting to note that (as demonstrated before and exemplified by marker D22S430 in Fig. 1d) the meningioma of our patient has no LOH 22 and the retained INII allele carries no additional inactivating mutation [2], indicating that this tumour is not the result of inheritance of the mutated INII gene. In contrast, the meningioma displayed genetic changes that are characteristic for radiation-induced meningiomas $[1,7,9]$, such as a complex karyotype, as evidenced by the SNP analysis (Table 1), including extensive losses on chromosome arm $1 \mathrm{p}$, but no loss of chromosome 22, and no mutation in the NF2 gene [2]. The genetic changes found in the meningioma proved not to be present in the AT/RT or myoepithelioma (Fig. 1a-c, and data not shown), demonstrating that the meningioma is genetically not related to these tumours and should not be considered as a late and more differentiated recurrence or metastasis of the AT/RT.

In conclusion, the clinical and genetic characteristics of the meningioma clearly indicate that the tumour developed as a consequence of the radiation therapy. Recent evidence suggests that long-term survival of patients with a childhood AT/RT can occur especially after aggressive treatment including radiotherapy $[10,12]$. The possible late effects of radiation therapy have been considered as serious drawbacks of this treatment. This is the first case in literature in which such a possible consequence in the form of a radiation-induced meningioma is actually documented in a long-term survivor of an AT/RT. Compared to sporadic meningiomas, radiation-induced meningiomas are more aggressive and have a higher chance to recur [1]. Because of this unfavourable prognosis, continuous medical surveillance of our patient is warranted.

Acknowledgement We gratefully acknowledge the financial support from the Dutch Cancer Society, grants KUN 2003-2975 (PW) and UVA 2001-2561 (TH)

Open Access This article is distributed under the terms of the Creative Commons Attribution Noncommercial License which permits any noncommercial use, distribution, and reproduction in any medium, provided the original author(s) and source are credited.

\section{References}

1. Al Mefty O, Topsakal C, Pravdenkova S, Sawyer JR, Harrison MJ (2004) Radiation-induced meningiomas: clinical, pathological, cytokinetic, and cytogenetic characteristics. J Neurosurg 100:1002-1013

2. Ammerlaan ACJ, Ararou A, Houben MPWA, Baas F, Tijssen CC, Teepen JLJM, Wesseling P, Hulsebos TJM (2007) Long-term survival and transmission of INI1-mutation via nonpenetrant males in a family with rhabdoid tumour predisposition syndrome. Br J Cancer, DOI 10.1038/sj.bjc.6604156

3. Bijlsma EK, Voesten AM, Bijleveld EH, Troost D, Westerveld A, Merel P, Thomas G, Hulsebos TJM (1995) Molecular analysis of genetic changes in ependymomas. Genes Chromosomes Cancer $13: 272-277$

4. Harrison MJ, Wolfe DE, Lau TS, Mitnick RJ, Sachdev VP (1991) Radiation-induced meningiomas: experience at the Mount Sinai Hospital and review of the literature. J Neurosurg 75:564-574

5. Hulsebos TJ, Plomp AS, Wolterman RA, Robanus-Maandag EC, Baas F, Wesseling P (2007) Germline mutation of INI1/SMARCB1 in familial schwannomatosis. Am J Hum Genet 80:805-810

6. Janson K, Nedzi LA, David O, Schorin M, Walsh JW, Bhattacharjee M, Pridjian G, Tan L, Judkins AR, Biegel JA (2006) Predisposition to atypical teratoid/rhabdoid tumor due to an inherited INI1 mutation. Pediatr Blood Cancer 47:279-284

7. Ragel BT, Jensen RL (2005) Molecular genetics of meningiomas. Neurosurg Focus 19:E9

8. Schmitz U, Mueller W, Weber M, Sevenet N, Delattre O, von Deimling A (2001) INI1 mutations in meningiomas at a potential hotspot in exon 9. Br J Cancer 84:199-201

9. Shoshan Y, Chernova O, Juen SS, Somerville RP, Israel Z, Barnett GH, Cowell JK (2000) Radiation-induced meningioma: a distinct molecular genetic pattern. J Neuropathol Exp Neurol 59:614-620

10. Squire SE, Chan MD, Marcus KJ (2007) Atypical teratoid/ rhabdoid tumor: the controversy behind radiation therapy. J Neurooncol 81:97-111

11. Taylor MD, Gokgoz N, Andrulis IL, Mainprize TG, Drake JM, Rutka JT (2000) Familial posterior fossa brain tumors of infancy secondary to germline mutation of the hSNF5 gene. Am J Hum Genet 66:1403-1406

12. Tekautz TM, Fuller CE, Blaney S, Fouladi M, Broniscer A, Merchant TE, Krasin M, Dalton J, Hale G, Kun LE, Wallace D, Gilbertson RJ, Gajjar A (2005) Atypical teratoid/rhabdoid tumors (ATRT): improved survival in children 3 years of age and older with radiation therapy and high-dose alkylator-based chemotherapy. J Clin Oncol 23:1491-1499 